\title{
Conhecimento e Atitude: perfil de pessoas com diabetes em diálise
}

\author{
Knowledge and attitude: profile of diabetics in dialysis \\ Conocimiento y actitud: perfil de personas con diabetes en diálisis
}

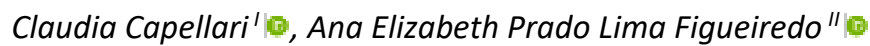

'Faculdades Integradas de Taquara, Taquara, RS, Brasil; "Pontifícia Universidade Católica do Rio Grande do Sul, Porto Alegre, RS, Brasil

\section{RESUMO}

Objetivo: identificar o conhecimento e o enfrentamento do diabetes junto a pessoas com diabetes em diálise. Método: estudo transversal, realizado com pacientes com diabetes tipo 2. Os instrumentos utilizados foram o Diabetes Knowledge Scale Questionnaire (DKN-A) e o Diabetes Attitude Questionnaire (ATT-19). Resultados: participaram 71 pacientes, com idade média de $61,81 \pm 14,93$ anos. A retinopatia diabética foi a complicação prevalente $(81,69 \%)$; hipertensão arterial sistêmica foi a comorbidade (83,09\%). A glicemia em jejum apresentou mediana de $152(124-228,5) \mathrm{mg} / \mathrm{dl}$ e a hemoglobina glicada de 7,5 $(6,42-8,27) \mathrm{mg} / \mathrm{dl}$. O DKN-A apresentou escore médio de $7,84 \pm 2,55$ pontos; seu item com maior número de acertos foi a conduta em caso de hipoglicemia; enquanto o com menor número de acertos foi em relação à cetonúria e substituições alimentares. 0 instrumento ATT-19 obteve média de 50,26 $\pm 11,7$ pontos. Conclusão: pessoas com diabetes, em diálise, apresentam conhecimento deficiente em relação ao diabetes, assim como baixo enfrentamento da doença.

Descritores: Insuficiência Renal Crônica; Diabetes Mellitus; Enfermagem em Nefrologia; Educação em Saúde; Conhecimento.

\begin{abstract}
Objective: to identify knowledge of, and coping with, diabetes mellitus among diabetics undergoing dialysis. Method: in this cross-sectional study of patients with type 2 diabetes, the instruments used were the Diabetes Knowledge Scale Questionnaire (DKN-A) and Diabetes Attitude Questionnaire (ATT-19). Results: mean age of the 71 participants was $61.81 \pm 14.93$ years. The most prevalent complication was diabetic retinopathy $(81.69 \%)$, and the most prevalent comorbidity was systemic arterial hypertension (83.09\%). Median fasting glycemia and glycated hemoglobin were 152 (124-228.5) mg/dl and 7,5 (6,42-8,27) $\mathrm{mg} / \mathrm{dl}$, respectively. Average DKN-A score was $7.84 \pm 2.55$; the highest success rate was on how to respond to hypoglycemia; the lowest was about ketones in urine and substitute foods. Mean ATT-19 score was $50.26 \pm 11.7$. Conclusion: the diabetics in dialysis showed deficient knowledge of diabetes and had negative attitudes to the disease.

Descriptors: Kidney Failure, Chronic; Diabetes Mellitus; Nephrology Nursing; Health Education; Knowledge.
\end{abstract}

\section{RESUMEN}

Objetivo: identificar el conocimiento y el afrontamiento de la diabetes mellitus entre los diabéticos en diálisis. Método: en este estudio transversal de pacientes con diabetes tipo 2, los instrumentos utilizados fueron el Diabetes Knowledge Scale Questionnaire (DKN-A) y Diabetes Attitude Questionnaire (ATT-19). Resultados: la edad media de los 71 participantes fue de $61,81 \pm 14,93$ años. La complicación más prevalente fue la retinopatía diabética $(81,69 \%)$ y la comorbilidad más prevalente fue la hipertensión arterial sistémica $(83,09 \%)$. La mediana de la glucemia en ayunas y la hemoglobina glucosilada fueron 152 (124$228,5) \mathrm{mg} / \mathrm{dl}$ y $7,5(6,42-8,27) \mathrm{mg} / \mathrm{dl}$, respectivamente. La puntuación promedio de DKN-A fue de 7,84 $\pm 2,55$; la tasa de éxito más alta fue sobre cómo responder a la hipoglucemia; el más bajo fue sobre las cetonas en la orina y los alimentos sustitutos. La puntuación media de ATT-19 fue 50,26 $\pm 11,7$. Conclusión: los diabéticos en diálisis mostraban un conocimiento deficiente de la diabetes y actitudes negativas hacia la enfermedad.

Descriptores: Fallo Renal Crónico; Diabetes Mellitus; Enfermería en Nefrología; Educación en Salud; Conocimento.

\section{INTRODUÇÃO}

O Diabetes Mellitus (DM) é uma das mais frequentes doenças crônicas do mundo, e pode afetar cerca de 425 milhões de pessoas ${ }^{1}$. No Brasil, a patologia atinge em torno de 7,6\% da população, o que representa mais de 15 milhões de indivíduos².

Está bem descrito que o adequado controle glicêmico é fundamental para a redução dos danos e complicações crônicas causados pela doença. Grandes estudos mostram que os níveis glicêmicos são diretamente proporcionais às complicações decorrentes do $\mathrm{DM}^{3,4}$. Neste sentido, a manutenção de níveis aceitáveis de glicose tem revelado benefícios em relação às complicações, em especial aquelas associadas à microvasculopatia e neuropatia 3,5,6.

Uma das consequências mais críticas do diabetes é a Doença Renal Crônica (DRC). Para as pessoas com ambas as patologias, as recomendações para o controle glicêmico fazem parte de uma estratégia de intervenções multifatorial, a fim de prevenir lesões microvasculares e outras injúrias ${ }^{7,8}$. 
Para o sucesso do tratamento é importante que o paciente participe do processo de cuidar. Dessa forma, acreditase que o conhecimento da doença e suas alternativas de controle sejam essenciais para minimizar eventos adversos e melhorar a qualidade de vida. Nesse contexto, a educação em saúde surge como potencial para melhoria no empoderamento do paciente. A partir do exposto, infere-se que os profissionais são capazes de atuar como motivadores, facilitadores e promotores da conscientização dos indivíduos, contribuindo para a adesão ao tratamento, para o desenvolvimento da capacidade de autocuidado e mudança de estilo de vida ${ }^{9,10}$.

Nesse contexto, esse estudo teve como objetivo identificar o conhecimento e atitudes de pessoas com diabetes e doença renal crônica em diálise.

\section{REVISÃO DA LITERATURA}

Para os profissionais de saúde, é importante ter consciência de quão bem os pacientes entendem sua patologia e sua atitude em relação a ela. Esses dados podem orientar estratégias e diretrizes de educação em saúde para a adesão ao tratamento. Embora existam poucos estudos sobre a população foco deste estudo, um estudo de caso recomenda intervenções planejadas pelo enfermeiro ${ }^{11}$.

Nesse sentido, alguns instrumentos podem subsidiar a tomada de decisão dos profissionais, fornecendo as informações necessárias sobre o conhecimento do paciente acerca de sua própria doença e as estratégias utilizadas por ele para enfrentá-la. Dois dos questionários são o Diabetes Knowledge Questionnaire (DKN-A) ${ }^{12,13}$ e Diabetes Attitude Questionnaire (ATT-19) ${ }^{13,14}$, validados para a realidade brasileira.

O DKN-A é composto por 15 questões de múltipla escolha e abrange aspectos relacionados ao conhecimento geral sobre diabetes mellitus. As perguntas são sobre fisiologia básica, grupos de alimentos e manejo do DM. A escala de medição utilizada é de 0 a 15. É atribuída uma pontuação de um (1) à resposta correta e zero (0) para a incorreta. Escore superior a oito (8) indica conhecimento suficiente sobre diabetes mellitus ${ }^{15}$.

O ATT-19 é um instrumento que busca a medida do ajuste psicológico do diabetes mellitus, desenvolvido em resposta às necessidades de avaliação dos aspectos psicológicos e emocionais da doença. Ele contém dezenove itens que incluem seis fatores: a) estresse associado ao DM, b) receptividade ao tratamento, c) confiança no tratamento, d) eficiência pessoal, e) percepção da saúde, f) aceitação social, cujas respostas são medidas usando cinco pontos em uma escala Likert (discordo completamente - escore 1; até concordar completamente - escore 5). O valor total da pontuação pode variar de 19 a 95 pontos. Escore superior a 70 indica atitude positiva em relação à doença ${ }^{15}$. Para esse instrumento, a atitude está relacionada à decisão do indivíduo de adotar ou não as medidas de autocuidado para o controle do diabetes ${ }^{16}$.

Esses questionários foram citados em uma série de estudos que medem o impacto de intervenções em pessoas com diabetes, principalmente aquelas que envolvem educação em saúde, ou investigam conhecimento, enfrentamento e autocuidado como dados subsidiários para as ações da equipe de saúde ${ }^{16-25}$. Assim, avaliar o conhecimento e os aspectos emocionais é importante para fornecer cuidados e informações consistentes com o público atendido.

\section{MÉTODO}

Trata-se de um estudo transversal, aprovado pelo Comitê de Ética e Pesquisa da Pontifícia Universidade Católica do Rio Grande do Sul (PUCRS) sob o número Certificado de Apresentação para Apreciação Ética (CAAE) 51381215.4.0000.5336.

O estudo ocorreu em dois serviços de diálise no estado do Rio Grande do Sul, Brasil. Os dados foram coletados entre novembro de 2016 e abril de 2017.

A população foi constituída por pessoas com diabetes em diálise (hemodiálise e diálise peritoneal), vinculados aos serviços mencionados, totalizando 118 pessoas. Na amostra, foram incluídos os diabéticos tipo 2, maiores de 18 anos, alfabetizados, que aceitaram participar do estudo mediante assinatura do termo de consentimento livre e esclarecido.

Foram excluídos pacientes com deficiência grave não corrigida na audição, fala e amaurose total ou com doença neurológica degenerativa, relatados em prontuários. Também todos aqueles com menos de 20 pontos no Mini Exame do Estado Mental (MEEM) ${ }^{26}$. A seleção da amostra seguiu o diagrama apresentado na Figura 1.

Os pacientes que atenderam aos critérios de inclusão foram convidados a participar e, aqueles que aceitaram, assinaram o termo de consentimento livre e esclarecido e responderam ao MEEM.

Para aqueles com pontuação igual ou superior a 20 no MEEM, foi aplicado um instrumento para a coleta das variáveis sociodemográficas e clínicas, que incluíram idade, cor sexo, situação conjugal, escolaridade, renda, distância da residência em relação ao serviço de diálise, tempo de diagnóstico de diabetes, medicações, complicações crônicas e comorbidades, valores laboratoriais de glicemia em jejum, hemoglobina glicada, colesterol e triglicerídeos. Os dados 
sociodemográficos foram coletados à beira leito, durante uma sessão de diálise, pela pesquisadora. Os dados clínicos foram obtidos por consulta ao prontuário.

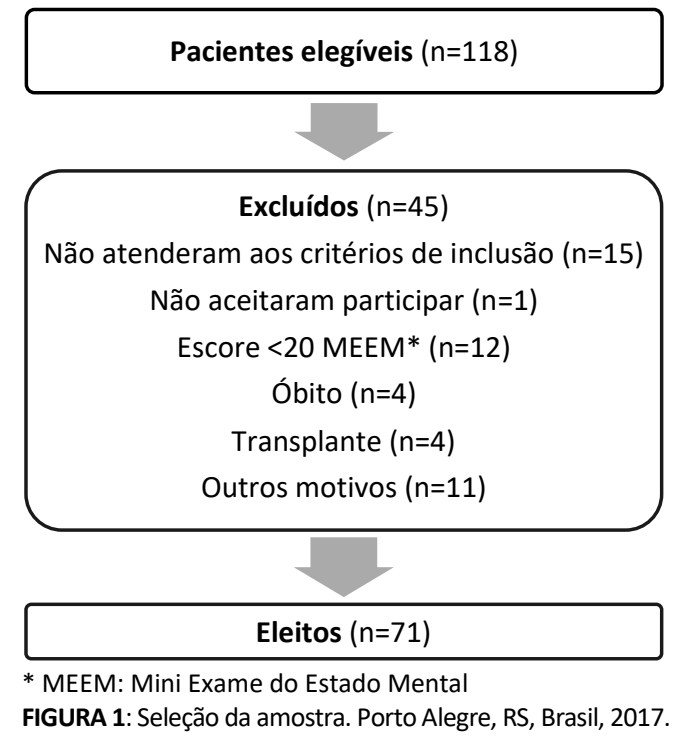

Na mesma ocasião da coleta dos dados sociodemográficos, os participantes responderam aos questionários DKN-A e ATT-19 ${ }^{15}$. Para tanto, foi utilizado o aplicativo Survey Monkey ${ }^{\circledR}$, via tablet com acesso à internet. Os próprios pacientes, com o equipamento oferecido pela pesquisadora, responderam aos questionários, após explicação de como preenchê-los.

Se procedeu a extração dos dados diretamente do software Survey Monkey, na forma de planilha Excel. Após organização das planilhas, foi realizada a análise estatística por meio do software Statistical Package for the Social Sciences (SPSS), versão 20.0. A apresentação dos resultados ocorreu pela estatística descritiva - distribuição absoluta e relativa ( $\mathrm{n}-\%)$, bem como, pelas medidas de tendência central e de variabilidade (média \pm desvio padrão, mediana (10 - 3o quartil). O intervalo de confiança foi de $95 \%(p<0,05)$.

\section{RESULTADOS}

Participaram do estudo 71 pacientes, com idade média de 61,81£14,93 anos, 62 (87,32\%) eram brancos, 37 $(52,11 \%)$ eram do sexo masculino e $37(52,11 \%)$ eram casados ou em um relacionamento estável. Entre os participantes, o tempo médio de escolaridade foi de $5(3,5-8,5)$ anos; 6 (8,45\%) possuíam diploma universitário. Sessenta e nove (69) participantes eram aposentados ou recebiam pensões do Instituto Nacional de Seguridade Social (INSS) e a renda familiar média foi de 3,22 (2-4) salários mínimos; 2 (2,81\%) participantes exerciam atividade laboral. A distância média do centro de diálise até a residência do participante foi de 19,25 $(5-24,5) \mathrm{km}$.

O tempo médio desde o diagnóstico de Diabetes Mellitus foi de 17,88 (10-25) anos e para a DRC 4 (2-8) anos. Sobre os antidiabéticos, 53 (74,64\%) participantes relataram a aplicação de insulina associada ao controle da dieta como forma de tratamento do DM. Nenhum fazia uso de hipoglicemiantes orais.

Em relação às complicações crônicas, além da DRC, que afeta $100 \%$ da população estudada, foi identificada retinopatia diabética em 58 (81,69\%), neuropatia em 48 (67,60\%), pé diabético em 10 (14,08\%), vasculopatia em 23 (32,39\%), doença vascular cerebral em 17 (23,94\%), doença coronariana em 29 (40,84\%) e amputação por diabetes mellitus em 9 (12,67\%).

Sobre as comorbidades e fatores de risco, foram observadas hipertensão arterial em 59 (83,09\%) participantes, dislipidemia em 32 (45,07\%), obesidade em 24 (33,8\%), sedentarismo em 41 (57,74\%) e tabagismo em 6 (8,45\%).

Os valores medianos para glicemia em jejum e hemoglobina glicada foram $152(124-228,5)$ mg/dl e 7,5 (6,42-8,27) $\mathrm{mg} / \mathrm{dl}$, respectivamente.

Em relação aos valores totais de colesterol, a média foi de $167 \pm 43,9 \mathrm{mg} / \mathrm{dl}$. Já os triglicerídeos apresentaram mediana de 157 (108,5-235) mg/dl e, para 38 (53,52\%) participantes, esses valores estavam acima de $150 \mathrm{mg} / \mathrm{dl}$.

A respeito do conhecimento sobre o diabetes, a Figura 2 mostra a dispersão das pontuações do questionário DKNA. O escore médio foi de 7,84 $\pm 2,55$ pontos, sendo o escore superior a 8 alcançado por 24 (33,80\%) participantes. 


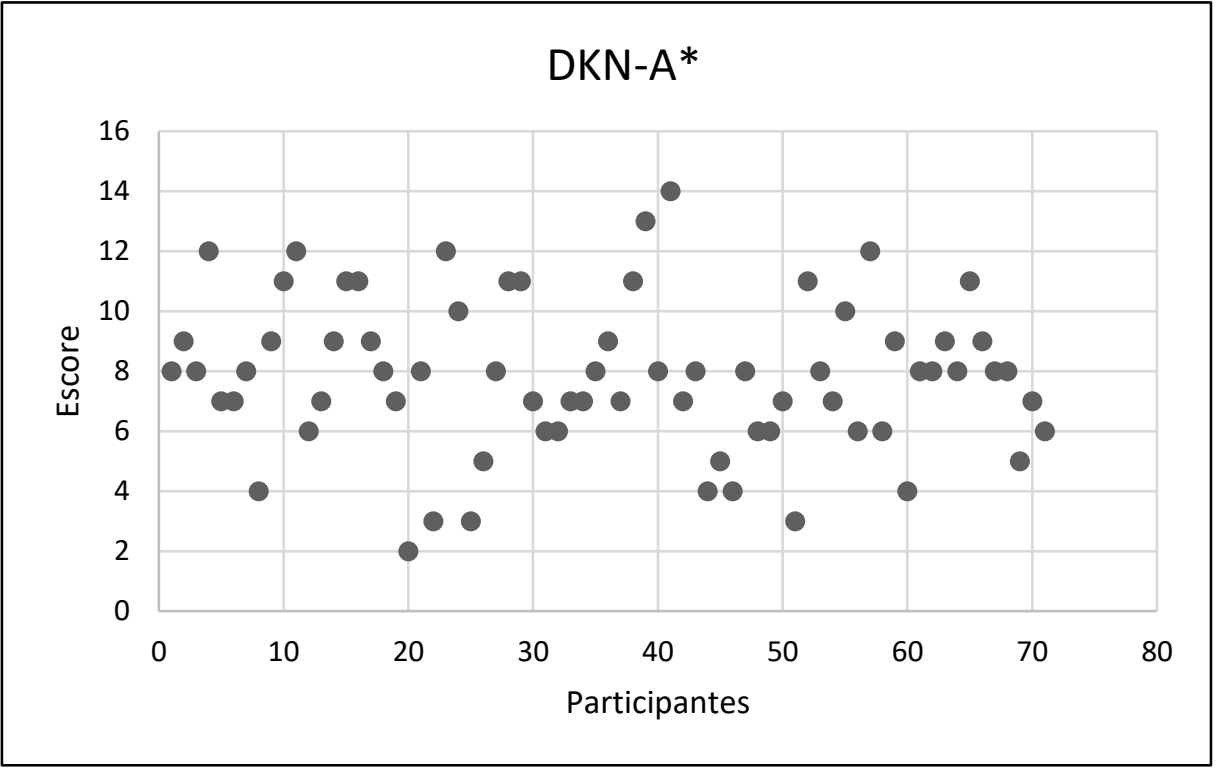

FIGURA 2: Dispersão dos escores do questionário Diabetes Knowledge Scale Questionnaire (DKN-A*). Porto Alegre, RS, Brasil, 2017.

Na Tabela 1 são apresentadas as questões do DKN-A, agrupadas de acordo com os atributos e a percentagem de respostas corretas e incorretas para cada questão.

TABELA 1: Distribuição absoluta e relativa das classificações do instrumento DKN-A*, de acordo com o número de respostas corretas e incorretas, agrupadas por atributos (Fisiologia básica, Grupos de alimentos e substituições, Manejo do $\mathrm{DM}^{\dagger}$ em caso de intercorrências e princípios gerais do cuidado com a doença). Porto Alegre, RS, Brasil, 2017.

\begin{tabular}{|c|c|c|c|c|c|}
\hline \multirow{2}{*}{ no } & \multirow{2}{*}{ Questão } & \multicolumn{2}{|c|}{ Correto } & \multicolumn{2}{|c|}{ Incorreto } \\
\hline & & $\mathbf{n}$ & $\%$ & $\mathbf{n}$ & $\%$ \\
\hline & Fisiologia básica & & & & \\
\hline 1 & $\begin{array}{l}\text { Indicação da variação de açúcar no sangue quando } \mathrm{DM}^{+} \text {está descontrolado } \\
\text { (normal / alto / baixo) }\end{array}$ & 56 & 78,87 & 15 & 21,13 \\
\hline 3 & Indicação da faixa de variação NORMAL da glicose do sangue & 52 & 73,23 & 19 & 26,77 \\
\hline 6 & Identificação da cetonúria & 4 & 5,63 & 67 & 94,37 \\
\hline 7 & Identificação de complicações decorrentes do $\mathrm{DM}^{+}$(olhos, rins, pulmões) & 51 & 71,83 & 20 & 28,17 \\
\hline \multirow[t]{2}{*}{12} & Causas da hipoglicemia & 17 & 23,94 & 54 & 76,06 \\
\hline & Grupos de alimentos e substituições & & & & \\
\hline 4 & Composição da manteiga & 41 & 57,74 & 30 & 42,26 \\
\hline 5 & Composição do arroz & 43 & 60,56 & 28 & 39,44 \\
\hline 11 & Alimentos que se pode ingerir à vontade & 44 & 61,97 & 27 & 38,03 \\
\hline 14 & $\begin{array}{l}\text { Substituiç̧̃̃es corretas (pão francês/biscoito, ovo/carne moída, } \\
\text { leite/suco de laranja, macarrão/sopa de legumes) }\end{array}$ & 23 & 32,39 & 48 & 67,61 \\
\hline \multirow[t]{2}{*}{15} & $\begin{array}{l}\text { Substituição correta do pão francês (biscoito água e sal, pão de queijo, fatia de } \\
\text { queijo, deixar pra lá) }\end{array}$ & 20 & 28,16 & 51 & 71,84 \\
\hline & $\begin{array}{l}\text { Manejo do } \mathrm{DM}^{\dagger} \text { em caso de intercorrências e princípios gerais do cuidado com } \\
\text { a doença }\end{array}$ & & & & \\
\hline 2 & $\begin{array}{l}\text { Identificação de complicações relacionadas ao DM (coma, glicosúria, } \\
\text { complicações tardias) }\end{array}$ & 45 & 63,38 & 26 & 36,32 \\
\hline 8 & Conduta em caso de hiperglicemia & 36 & 50,70 & 35 & 49,30 \\
\hline 9 & Conduta para aplicação de insulina em caso de adoecimento ou inapetência & 37 & 52,11 & 34 & 47,89 \\
\hline 10 & Conduta em caso de hipoglicemia & 58 & 81,69 & 13 & 18,31 \\
\hline 13 & Referência à unidade de medida - um quilo & 30 & 42,26 & 41 & 57,74 \\
\hline
\end{tabular}

*DKN-A: Diabetes Knowledge Scale Questionnaire; ${ }^{\dagger}$ DM: Diabetes Mellitus

As atitudes em relação ao diabetes podem ser observadas a partir dos valores obtidos no questionário ATT-19 e apresentados na Figura 3. Os escores variaram de 28 a 77 pontos, com média 50,26 $\pm 11,7$. Quatro (5,63\%) participantes apresentaram escore acima de 70 pontos. 


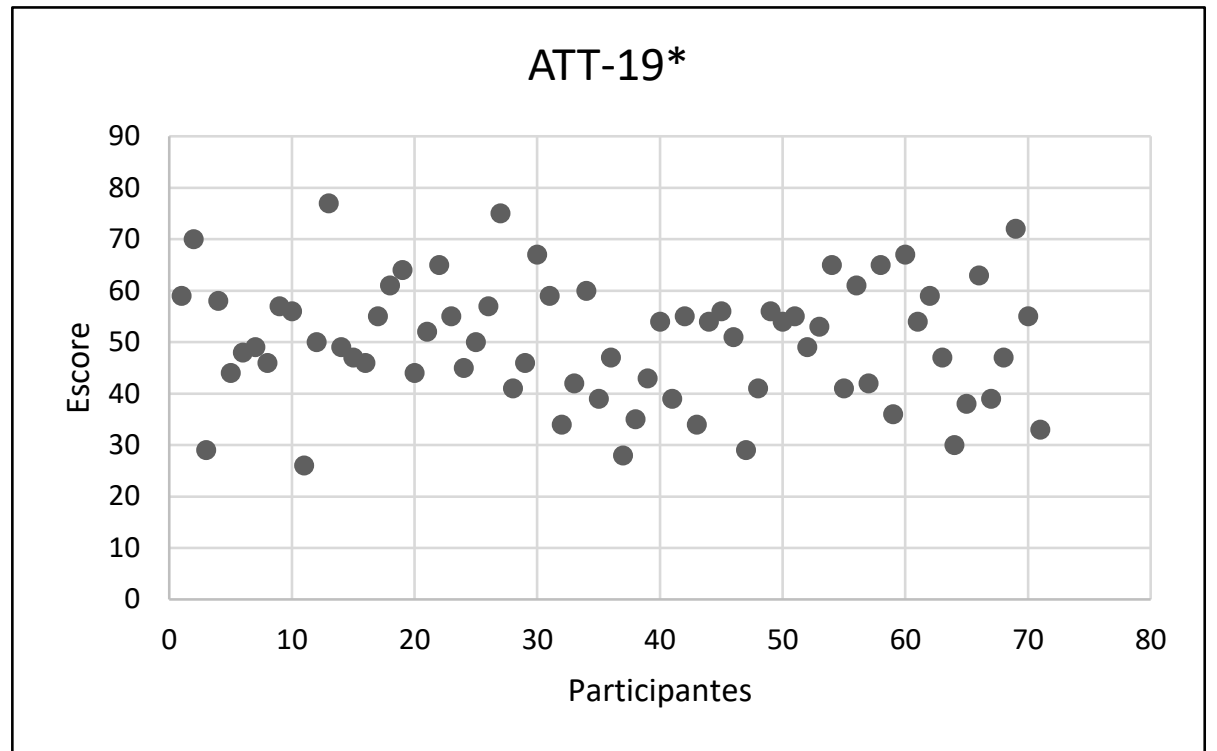

FIGURA 3: Dispersão dos escores do instrumento Diabetes Attitude Questionnaire (ATT-19*). Porto Alegre, RS, Brasil, 2017.

A Tabela 2 apresenta a distribuição das respostas do ATT-19, com base na escala Likert, que vai desde o Discordo Completamente até Concordo Completamente.

TABELA 2: Distribuição absoluta das respostas dos participantes de acordo com a escala Likert, do instrumento ATT-19*, agrupadas por atributos (Estresse associado ao diabetes, Receptividade ao tratamento, Crença no tratamento e Eficácia pessoal, Percepção sobre a saúde, Aceitação social). Porto Alegre, RS, Brasil, 2017.

\section{Questões}

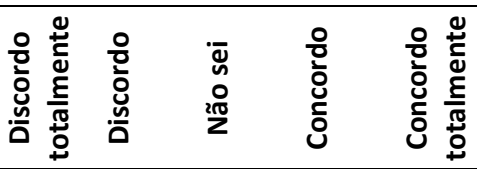

\section{Estresse associado ao Diabetes}

1. Se eu não tivesse DIABETE, eu seria uma pessoa bem diferente

3. Ter DIABETE foi a pior coisa que aconteceu na minha vida

4. A maioria das pessoas tem dificuldade em se adaptar ao fato de ter DIABETE

$\begin{array}{ccccc}24 & 37 & 1 & 5 & 4 \\ 29 & 18 & 0 & 17 & 7 \\ 13 & 37 & 5 & 16 & 0 \\ 2 & 5 & 0 & 35 & 29 \\ 8 & 38 & 1 & 14 & 10 \\ 4 & 27 & 1 & 19 & 20 \\ 4 & 15 & 0 & 39 & 13 \\ 4 & 8 & 2 & 31 & 26\end{array}$

5. Costumo sentir vergonha por ter DIABETE

8. O controle adequado da DIABETE envolve muito sacrifício e inconvenientes

10. Ser diagnosticado com DIABETE é o mesmo que ser condenado a uma vida de doença

11. Minha diabete não atrapalha muito minha vida social

19. Costumo achar que é injusto que eu tenha DIABETE e outras pessoas tenham uma saúde muito boa

\section{Receptividade ao tratamento}

7. Há pouca esperança de levar uma vida normal com DIABETE

15. DIABETE não é realmente um problema porque pode ser controlado

18. Acredito que convivo bem com a DIABETE

\section{Crença no tratamento}

2. Não gosto que me chamem de diabético

6. Parece que não tem muita coisa que eu possa fazer para controlar a minha DIABETE

9. Procuro não deixar que as pessoas saibam que tenho DIABETE

17. Não há ninguém com que meu possa falar abertamente sobre a minha DIABETE

\section{Eficácia pessoal}

12. Em geral, os medicos precisam ser muito mais atenciosos ao tartar as pessoas com DIABETE

13. Ter DIABETE durante muito tempo muda a personalidade da pessoa

\section{Percepção sobre a saúde}

14. Tenho dificuldade em saber se estou bem ou doente

\section{Aceitação social}

16. Não há nada que você possa fazer, se você tiver DIABETE

ATT-19: Diabetes Attitude Questionnaire 


\section{DISCUSSÃO}

Este estudo apresenta resultados de pacientes com diabetes em diálise. Outras investigações foram realizadas com pessoas com diabetes, mas há uma lacuna relacionada às pessoas que também têm DRC e realizam diálise. Nesse contexto, é importante observar o conhecimento e a atitude, acerca da doença, dos pacientes envolvidos.

Na presente investigação, os participantes possuíam idade média semelhante à de outros estudos que utilizaram os mesmos questionários de conhecimento e atitudes sobre $\mathrm{DM}^{27-30}$. No entanto, diferentemente desses, a maioria dos participantes da presente pesquisa é do sexo masculino. Uma explicação plausível para isso é o fato de o estudo ter sido realizado com pacientes em diálise, em que o número de homens prevalece em relação ao número de mulheres (58\% do sexo masculino) $)^{31}$.

Os dados relacionados ao nível de escolaridade corroboram os achados de outros estudos, que também indicam baixos níveis de educação formal ${ }^{25,29,32,33}$. Há evidências de que o nível de escolaridade não está relacionado a um maior conhecimento sobre o DM ou a uma atitude positiva para lidar com ele ${ }^{32}$. No entanto, a escolaridade pode interferir no entendimento das instruções fornecidas, mecanismos relacionados à doença e adesão ao tratamento. Esses fatos constituem um desafio para os profissionais de saúde, que precisam elaborar estratégias para fortalecer as habilidades cognitivas, motoras e afetivas das pessoas com diabetes, para alcançar um controle satisfatório da doença ${ }^{29}$.

Além da baixa escolaridade, foi encontrada baixa renda familiar, mesmo superior à relatada por uma revisão sistemática $(1,5 \pm 2)^{34}$. Acredita-se que esse fato possa dificultar o acesso das pessoas a serviços básicos, com impacto direto na saúde, como alimentação, transporte, educação e moradia adequados.

Em relação ao emprego, outras pesquisas relataram taxas de ocupação de $47,7 \%{ }^{32}$ e $30,5 \%{ }^{17}$, embora não tenham sido realizadas entre pacientes em diálise. Por outro lado, no presente estudo, poucos participantes relataram estar empregados. As pessoas em diálise, além de apresentar comorbidades importantes, precisam adaptar sua rotina para frequentar o centro de diálise três vezes por semana, o que dificulta a entrada no mercado de trabalho, bem como a manutenção do emprego. Além disso, a distância da residência ao centro de diálise influenciaria a chance de trabalhar. Esse fato aponta para a necessidade de um horário flexível, pois as pessoas dispendem tempo de deslocamento, além daquele relacionado ao tratamento dialítico.

No que diz respeito ao tempo de patologia, os estudos publicados indicam uma grande variedade de anos, com $9.5 \pm 7.9^{32}, 11.18 \pm 8.64^{29},<10^{25,28}$ e $11 \pm 8^{27}$. O tempo mais longo do diagnóstico de DM apresentado neste estudo pode estar relacionado à amostra participante (pacientes com diabetes em diálise). A nefropatia diabética é a principal complicação microvascular do diabetes, bem como a principal causa de DRC no mundo ${ }^{35}$ e, no Brasil, corresponde à causa primária de $31 \%$ dos pacientes em diálise ${ }^{36}$.

Em relação às complicações crônicas, a microvasculopatia está fortemente associada ao diabetes ${ }^{37}$, quando ocorre um desequilíbrio entre fatores pró-sobrevivência e mediadores inflamatórios. Neste estudo, além da insuficiência renal, a retinopatia diabética teve a maior prevalência, diferente dos resultados encontrados em um estudo realizado na Atenção Básica, que encontrou $15 \%$ de retinopatia ${ }^{38}$. Além dos impactos em termos de sofrimento humano, as complicações decorrentes do diabetes afetam diretamente o custo para os sistemas de saúde ${ }^{37}$. A comorbidade mais frequente foi a hipertensão, o que corrobora o indicador publicado pela Sociedade Brasileira de Nefrologia, que aponta a hipertensão como a principal causa de DRC no Brasil ${ }^{36}$.

O presente estudo mostra menos obesidade em comparação com um estudo com idosos, no qual 51\% apresentavam sobrepeso ${ }^{25}$. Por outro lado, os valores foram semelhantes aos encontrados na Atenção Básica Brasileira $(31,1 \%)^{38}$. Possivelmente, os participantes deste estudo, em tratamento dialítico, apresentam redução de peso, pois a doença renal em estágio terminal está associada ao aumento de citocinas inflamatórias e pode resultar em caquexia, com perda de reservas musculares e gordurosas ${ }^{39}$.

A respeito da glicemia, verificou-se que os níveis de hemoglobina glicada denotaram um controle glicêmico deficiente. Um estudo realizado com 353 pessoas detectou mediana da glicose em jejum de 157 (58-391) mg/dL e HbA1C of $8,67 \%(3,70-16,10 \%)^{38}$. Um estudo de referência mostrou que o controle intenso do diabetes impede a progressão das complicações do diabetes ${ }^{3}$. Esses dados indicam a importância de estabelecer um plano multidisciplinar de educação em saúde para melhor adesão ao tratamento e minimização das complicações decorrentes do diabetes.

Ao investigar o conhecimento sobre diabetes, o presente estudo corrobora os achados que identificaram que $66,67 \%$ e $64,6 \%$ dos participantes apresentaram escores $\leq 8$ no $D K N-A^{16,40}$. Escores abaixo de oito indicam conhecimento insuficiente sobre a doença.

Por outro lado, um estudo realizado com usuários de um programa de educação para o autocuidado em diabetes ${ }^{17}$ constatou que $78,05 \%$ dos participantes obtiveram pontuação acima de 8 , indicando conhecimento e compreensão 
adequados da doença. Também em estudo com pacientes da Estratégia de Saúde da Família, participantes de um grupo de pessoas com diabetes ${ }^{27}$, apontaram $81,5 \%$ dos indivíduos com mais de oito pontos no questionário DKN-A.

Entende-se que os dois últimos estudos, com resultados positivos em relação ao conhecimento sobre a doença, estão possivelmente relacionados às iniciativas educacionais realizadas pelas equipes de saúde. Essa percepção é validada pelos achados de outras pesquisas ${ }^{33,41}$, que obtiveram diferenças estatisticamente significantes no conhecimento sobre diabetes, comparando os resultados antes e depois de um programa educacional. O mesmo foi encontrado em outra investigação ${ }^{42}$, em que os autores concluíram que a educação se apresenta como uma estratégia eficaz, que contribui para que a pessoa com diabetes viva melhor com sua condição. Por fim, uma revisão sistemática sugere que a educação em saúde está relacionada a uma resposta positiva, ao comparar parâmetros fisiológicos, psicológicos e educacionais, resultados iniciais e finais dos estudos ${ }^{34}$.

No questionário DKN-A, as questões que obtiveram o maior número de respostas corretas corroboram dos achados de uma pesquisa, que encontrou $83,8 \%$ de acertos nas perguntas relacionadas à faixa de variação glicêmica quando DM descontrolado e $86 \%$ em relação à faixa normal de variação da glicose sanguínea ${ }^{27}$. Em relação às complicações decorrentes do DM e ao comportamento em relação à hipoglicemia, o mesmo estudo apontou 67,6\% e $60,3 \%$ de respostas corretas, respectivamente. O comportamento correto em caso de hipoglicemia é importante, pois faz com que o indivíduo retorne às suas funções rotineiras, superando o mal-estar instalado devido à ocorrência.

Em relação às questões com menor número de respostas corretas, foram as de número 6 (relacionada cetonas urinárias), 12 (causas de hipoglicemia) e 15 (substituição de pão francês), com respostas corretas de 5,63\%, 23, 94\% e $28,16 \%$, respectivamente. Quando comparado a outros estudos, o percentual de acertos foi de $39,2 \%, 27 \%$ e $39,9 \%$ / $12,6 \%$, respectivamente ${ }^{27}$. No mesmo ponto, estudo realizado com idosos encontra $19,8 \%, 11,4 \%$ e $1 \%$, respectivamente ${ }^{25}$. Os achados do presente estudo são consistentes com a realidade brasileira, uma vez que o teste de cetona na urina não é uma prática comum orientada nos serviços de saúde. A baixa taxa de respostas corretas sobre as causas da hipoglicemia indica a falta de entendimento que os participantes têm sobre sua doença, o que pode impactar o manejo da mesma, bem como as complicações inerentes ao estado de saúde. Também foram obtidos indicadores insatisfatórios no grupo de questões relacionadas ao manejo do DM e princípios gerais do tratamento da doença.

A lacuna de conhecimento sobre os grupos de alimentos e a substituição correta ficou evidente nos resultados descritos na Tabela 1. Os achados indicam necessidade urgente de iniciativas de educação em saúde a esta população. Enfermeiras, por exercerem suas atividades em diferentes contextos, têm muitas oportunidades de desenvolver este trabalho.

Autores apontam que a identificação do conhecimento sobre DM é um recurso relevante para direcionar a equipe multiprofissional a tomar decisões clínicas e propor plano terapêutico ${ }^{27}$. Outros autores defendem a necessidade de buscar novas estratégias educacionais, que permitam ao paciente diabético adquirir conhecimentos para o manejo da doença, incorporando-o ao seu cotidiano, com possibilidades de transformar suas atitudes em relação ao diabetes ${ }^{29}$. Uma revisão sobre intervenções educacionais e controle glicêmico concluiu que as práticas de educação em saúde para o controle e tratamento do diabetes, em todo o mundo, indicam o envolvimento dos profissionais de saúde na instrumentalização dos pacientes, com vistas à autonomia e à expansão do autocuidado, visando melhorar os resultados clínicos e a qualidade de vida ${ }^{43}$.

Em relação às atitudes no que se refere à patologia, o presente estudo encontrou resultados que identificaram uma baixa disponibilidade para enfrentar a doença, o que corrobora os achados de outras publicações: $95,56 \%{ }^{29} ; 52,7 \%$ em mulheres e $50,7 \%$ em homens ${ }^{17}, 93,7 \%^{28}, 99,4 \%^{32}$ e $85,9 \%$ em idosos ${ }^{25}$. Os resultados obtidos no ATT-19 estão de acordo com estudos que identificaram uma média de 63,23 pontos $^{32}, 67,90 \pm 8.0^{44}, 55,5 \pm 8.0^{32} 2$ e 58 (52-65) pontos ${ }^{25}$. Há evidências de que o conhecimento sobre patologia é diretamente proporcional às atitudes e ao autocuidado de pacientes diabéticos, em diálise ${ }^{45}$.

No resultado do ATT-19, observa-se que, embora os participantes considerem que o diabetes não perturba a vida social e que não é um problema; muitos relatam ter vergonha de ter a doença e não deixar que outros saibam sobre a patologia. Além disso, parece haver alguma resignação com a doença, quando apontam que nada pode ser feito se você tem diabetes, ao passo que muitos consideram injusto ter diabetes enquanto outras pessoas têm uma saúde muito boa. Embora as iniciativas de educação do paciente sejam necessárias e enfatizadas pela Associação Brasileira de Diabetes e pela Associação Americana de Diabetes, é necessário que os profissionais de saúde desenhem estratégias para aumentar o empoderamento dos pacientes, incentivando-os a assumir a liderança ${ }^{43}$, uma vez que a expansão do conhecimento não implica, necessariamente, em mudanças comportamentais necessárias para o controle da doença ${ }^{20}$.

Nesse sentido, acredita-se que o tratamento do diabetes exija diversas atividades de autocuidado, e os aspectos emocionais e cognitivos estão diretamente relacionados à capacidade de autocuidado e autogestão do diabetes. No entanto, os efeitos positivos da educação em saúde são fortemente prejudicados quando o paciente sofre de depressão ou angústia relacionada ao diabetes. Assim, a equipe de saúde precisa estar atenta aos aspectos psicológicos dos 
pacientes e, se necessário, o apoio psicológico deve ser integrado ao cuidado dessas pessoas ${ }^{46}$. 0 envolvimento dos pacientes em seu próprio cuidado contribui fortemente para o sucesso do tratamento, mas muitas vezes o incentivo e o apoio dos profissionais de saúde são necessários para que os pacientes encontrem motivação para cuidar de si ${ }^{47}$.

\section{Limitações do estudo}

Embora este estudo tenha produzido importantes achados relacionados ao conhecimento e atitudes relacionados ao diabetes naqueles que fazem diálise, algumas limitações são reconhecidas. O primeiro diz respeito ao número de participantes. Embora dois serviços de diálise tenham sido incluídos, há necessidade de estudos que incorporem um número maior de sujeitos, a fim de aumentar a capacidade de generalização. A segunda limitação é o desenho do estudo, transversal. Nesse sentido, são necessários estudos prospectivos, com intervenções em educação em saúde, para que os resultados possam melhor orientar a prática clínica.

\section{CONCLUSÕES}

Os participantes deste estudo demonstraram ter conhecimento insuficiente sobre diabetes. Esse fato tem impacto na capacidade de autocuidado, também influenciada pelos aspectos psicológicos, evidenciados aqui pelas atitudes em relação ao diabetes, que também se mostraram insatisfatórias na maioria da amostra.

Considerando que existe uma relação entre escolaridade, conhecimento da doença e estado de saúde, é compreensível que os indivíduos tenham diversos níveis de compreensão sobre sua patologia e, consequentemente, tenham diferentes estratégias de enfrentamento. Os processos de educação em saúde realizados na prática clínica, portanto, devem considerar o conhecimento dos pacientes sobre sua patologia, bem como os aspectos psicológicos e emocionais relacionados à doença.

Entre as estratégias utilizadas pelos enfermeiros, os questionários validados podem apoiar a educação em saúde como uma forma de adequar os cuidados, capacitar os pacientes e torná-los corresponsáveis por seu tratamento e por sua condição de saúde.

\section{REFERÊNCIAS}

1. Cho NH, Shaw JE, Karuranga S, Huang Y, da Rocha Fernandes JD, Ohlrogge AW, et al. IDF Diabetes Atlas: Global estimates of diabetes prevalence for 2017 and projections for 2045. Diabetes Res. Clin. Pract. 2018 [cited 2020 Feb 20]; 138:271-81. DOI: https://doi.org/10.1016/j.diabres.2018.02.023.

2. Brazil. Vigitel Brazil 2017: surveillance of risk and protective factors for chronic diseases by telephone survey: estimates of frequency and sociodemographic distribution of risk and protective factors for chronic diseases in the capitals of the 26 Brazilian states and the Federal District in 2017. Ministry-of-Health. Brasília. [cited 2020 Feb 20] Available from http://bvsms.saude.gov.br/bvs/publicacoes/vigitel_brasil_2017_vigilancia_fatores_riscos.pdf.

3. Nathan DM, Genuth S, Lachin J, Cleary P, Crofford O, Davis M, et al. The effect of intensive treatment of diabetes on the development and progression of long-term complications in insulin-dependent diabetes mellitus. N. Engl. J. Med. 1993 [cited 2020 Mar 10]; 329(14):977-86. DOI: https://doi.org/10.1056/NEJM199309303291401.

4. Writing Group for the DCCT/EDIC Research Group. Coprogression of cardiovascular risk factors in type 1 diabetes during 30 years of follow-up in the DCCT/EDIC study. Diabetes Care. 2016 [cited 2020 Mar 7]; 39(9):1621-30. DOI: https://doi.org/10.2337/dc16-0502.

5. Nathan DM, Buse JB, Davidson MB, Ferrannini E, Holman RR, Sherwin R, et al. Medical management of hyperglycemia in type 2 diabetes: a consensus algorithm for the initiation and adjustment of therapy: a consensus statement of the American Diabetes Association and the European Association for the Study of Diabetes. Diabetes Care. 2009 [cited 2020 Mar 10]; 32(1):193-203. DOI: https://doi.org/10.2337/dc08-9025.

6. Bem AF, Kunde J. The importance of glycated hemoglobin determination in the management of chronic complications associated with diabetes mellitus. .J Bras. Patol. Med. Lab. 2006 [cited 2020 Mar 10]; 42(3). DOI: https://doi.org/10.1590/S1676-24442006000300007.

7. Kirsztajn GM, Salgado Filho N, Draibe SA, Netto MVdP, Thome FS, Souza E, et al. Fast Reading of the KDIGO 2012: Guidelines for evaluation and management of chronic kidney disease in clinical practice. J. Bras. Nefrol. 2014 [cited 2020 Mar 8]: 63-73. DOI: https://doi.org/10.5935/0101-2800.20140012.

8. Eknoyan G, Lameire N, Eckardt K, Kasiske B, Wheeler D, Levin A, et al. KDIGO 2012 clinical practice guideline for the evaluation and management of chronic kidney disease. Kidney International. 2013 [cited 2019 Dez 10]; 3:5-14. DOI: https://doi.org/10.1038/kisup.2012.75.

9. Assunção TS, Ursine PGS. A study of factors associated to non-pharmacological treatment delivered by the Family Health Program in Ventosa, Belo Horizonte, to carriers of diabetes mellitus. Ciênc. Saúde Coletiva. 2008 [cited 2020 Mar 7]; 13(Suppl 2):2189-97. DOI: https://doi.org/10.1590/S1413-81232008000900024.

10. Davies MJ, Heller S, Skinner TC, Campbell MJ, Carey ME, Cradock S, et al. Effectiveness of the diabetes education and self management for ongoing and newly diagnosed (DESMOND) programme for people with newly diagnosed type 2 diabetes: cluster randomised controlled trial. BMJ. 2008 [cited 2020 Mar 7]; 336(7642):491-5. DOI: https://doi.org/10.1136/bmj.39474.922025.BE. 
11. Mascarenhas NB, Pereira A, Silva RSd, Silva MGd. Systematization of nursing assistance to patients with diabetes mellitus and chronic renal. Rev. Bras. Enferm. 2011 [cited 2020 Mar 7]; 64:203-8. Available from: https://www.scielo.br/scielo.php?script=sci_abstract\&pid=S0034-71672011000100031\&lng=en\&nrm=iso.

12. Beeney LJ, Dunn SM, Welch G. Measurement of diabetes knowledge: the development of the DKN scales. In: Harwood, Publishers A, eds. Handbook of psychology and diabetes. Amsterdam. 2001:159-189.

13. Torres HC, Hortale VA, Schall VT. Validation of Diabetes Mellitus knowledge (DKN-A) and attitude (ATT-19) questionnaires. Rev. Saúde Pública. 2005 [cited 2020 Feb 20]; 39(6):906-911. DOI: https://doi.org/10.1590/\$0034-89102005000600006.

14. Welch G, Dunn SM, Beeney LJ. The ATT39: A measure of psychological adjustment to diabetes. Amsterdam. 2001.

15. Torres HC, Hortale VA, Schall VT. Validation of Diabetes Mellitus knowledge (DKN-A) and attitude (ATT-19) questionnaires. Rev. Saúde Pública. 2005 [cited 2020 Feb 20]; 39(6):906-911. DOI: https://doi.org/10.1590/S0034-89102005000600006.

16. Rodrigues FFL, Santos MAd, Teixeira CRdS, Gonela JT, Zanetti ML. Relationship between knowledge, attitude, education and duration of disease in individuals with diabetes mellitus. Acta Paulista de Enfermagem. 2012 [cited 2020 Feb 20]; 25(2):284-90. DOI: https://doi.org/10.1590/S0103-21002012000200020.

17. Rodrigues FFL, Zanetti ML, Santos MAd, Martins TA, Sousa VD, Teixeira CRdS. Knowledge and attitude: important components in diabetes education. Revista Latino-Americana de Enfermagem. 2009 [cited 2020 Feb 20]; 17(4):468-73. DOI: https://doi.org/10.1590/S0104-11692009000400006.

18. de Oliveira KCS, Zanetti ML. Knowledge and attitudes of patients with diabetes mellitus in a primary health care system. Revista da Escola de Enfermagem da USP. 2011 [cited 2020 Feb 20]; 45(4). DOI: https://doi.org/10.1590/S0080-62342011000400010.

19. de Oliveira Santos BM, Caixeta ACM, da Silva AA, de Souza Teixeira CR. Knowledge and attitudes in diabetes mellitus type 2: subsidies for self-care and health promotion. Arquivos de Ciências da Saúde. 2016 [cited 2020 Feb 20] (4):31-36\%V 23. DOI: https://doi.org/10.17696/2318-3691.23.4.2016.443.

20. Pereira DA, Costa NMdSC, Sousa ALL, Jardim PCBV, Zanini CRdO. The effect of educational intervention on the disease knowledge of diabetes mellitus patients. Revista Latino-Americana de Enfermagem. 2012 [cited 2020 Feb 22];20(3):478-85. DOI: https://doi.org/10.1590/S0104-11692012000300008.

21. Gonçalves NEXM, Zanetti ML, Neiva CM, Vassimon HS. Knowledge of individuals with diabetes mellitus in the Family Health Strategy. Revista de Enfermagem UFPE online. 2017 [cited 2020 Feb 22]; 11(7):2779-2787. DOI: https://doi.org/10.5205/reuol.10939-97553-1-RV.1107201718.

22. Hussain Z, Yusoff ZM, Sulaiman SA. A study exploring the association of attitude and treatment satisfaction with glycaemic level among gestational diabetes mellitus patients. Prim. Care Diabetes. 2015 [cited 2020 Feb 22]; 9(4):275-82. DOI: https://doi.org/10.5205/reuol.10939-97553-1-RV.1107201718.

23. Campos SM, Aparecida DF, Santos Nascimento J, Dos Santos Tavares DM. Correlation of quality of life with knowledge and attitude of diabetic elderly. Invest. educ. enferm. 2016 [cited 2020 Feb 22]; 34(1):180-8. DOI: https://doi.org/10.17533/udea.iee.v34n1a20.

24. Figueira ALG, Boas LCGV, Coelho ACM, Freitas MCF, Pace AE. Educational interventions for knowledge on the disease, treatment adherence and control of diabetes mellitus. Rev. Lat. Am. Enfermagem. 2017 [cited 2020 Mar 9]; 25:e2863. DOI: https://doi.org/10.17533/udea.iee.v34n1a20.

25. Borba AKOT, Arruda IKG, Marques APO, Leal MCC, Diniz ADS. Knowledge and attitude about diabetes self-care of older adults in primary health care. Cien. Saude Colet. 2019 [cited 2020 Mar 9]; 24(1):125-36. DOI: https://doi.org/10.17533/udea.iee.v34n1a20.

26. Brucki SM, Nitrini R, Caramelli P, Bertolucci PH, Okamoto IH. Suggestions for using the mini-mental state exam in Brazil. Arq. Neuropsiquiatr. 2003 [cited 2020 Mar 8]; 61(3B):777-81. DOI: https://doi.org/10.1590/S0004-282X2003000500014.

27. Gonçalves NEXM, Zanetti ML, Neiva CM, Vassimon HS. Revista de enfermagem UFPE. 2017 [cited 2020 Mar 8]; 11(7):27792787. DOI: https://doi.org/10.1590/S0004-282X2003000500014.

28. Oliveira KCSd, Zanetti ML. Knowledge and attitudes of patients with diabetes mellitus in a primary health care system. Revista da Escola de Enfermagem da USP. 2011 [cited 2020 Mar 8]; 45(4):862-8. DOI: https://dx.doi.org/10.1590/S0080-62342011000400010.

29. Rodrigues FFL, Santos MAd, Teixeira CRdS, Gonela JT, Zanetti ML. Relationship between knowledge, attitude, education and duration of disease in individuals with diabetes mellitus. Acta Paulista de Enfermagem. 2012 [cited 2020 Mar 10]; 25(2):284-90. DOI: https://doi.org/10.1590/S0103-21002012000200020.

30. de Oliveira KCS, Zanetti ML. Knowledge and attitudes of patients with diabetes mellitus in a primary health care system. Revista da Escola de Enfermagem da USP. 2011 [cited 2020 Mar 10]; 45(4). DOI: https://doi.org/10.1590/S0080-62342011000400010.

31. Sesso RC, Lopes AA, Thomé FS, Lugon JR, Martins CT. Brazilian Chronic Dialysis Census 2014. Jornal Brasileiro de Nefrologia. 2016 [cited 2020 Mar 10]; 38(1):54-61. DOI: https://doi.org/10.5935/0101-2800.20170049.

32. de Oliveira Santos BM, Caixeta ACM, da Silva AA, de Souza Teixeira CR. Knowledge and attitudes towards diabetes mellitus type 2: contributions to self-care and health promotion. Arquivos de Ciências da Saúde. 2016 [cited 2020 Mar 10] (4):31-36\%V 23. DOI: https://doi.org/10.17696/2318-3691.23.4.2016.443.

33. Otero LM, Zanetti ML, Ogrizio MD. Knowledge of diabetic patients about their disease before and after implementing a diabetes education program. Revista Latino-Americana de Enfermagem. 2008 [cited 2020 Mar 10]; 16(2):231-7. DOI: https://doi.org/10.1590/S0104-11692008000200010. 
34. Iquize RCC, Theodoro FCET, Carvalho KA, Oliveira MdA, Barros JdF, Silva ARd. Educational practices in diabetic patient and perspective of health professional: a systematic review. Jornal Brasileiro de Nefrologia. 2017 [cited 2020 Mar 10]; 39(2):196204. DOI: https://doi.org/10.5935/0101-2800.20170034.

35. Moreira H, Sette J, Keiralla L, et al. Diabetes mellitus, hypertension and chronic kidney disease: treatment strategies and their limitations. Rev. Bras. Hipertens. 2008 [cited 2020 Mar 10]; 15(2):111-6. Available from: http://departamentos.cardiol.br/dha/revista/15-2/17-diabetes.pdf.

36. Thomé FS, Sesso RC, Lopes AA, Lugon JR, Martins CT. Brazilian chronic dialysis survey 2017. J. Bras. Nefrol. 2019 [cited 2020 Mar 11]; 41(2): 208-14. DOI: https://doi.org/10.1590/2175-8239-jbn-2018-0178.

37. Muc R, Saracen A, Grabska-Liberek I. Associations of Diabetic Retinopathy with Retinal Neurodegeneration on the Background of Diabetes Mellitus. Overview of Recent Medical Studies with an Assessment of the Impact on Healthcare systems. Open Med (Wars). 2018 [cited 2020 Mar 11]; 13:130-6. DOI: https://doi.org/10.1515/med-2018-0008.

38. Assunção SC, Fonseca AP, Silveira MF, Caldeira AP, Pinho Ld. Knowledge and attitude of patients with diabetes mellitus in Primary Health Care. Escola Anna Nery. 2017 [cited 2020 Mar 11]; 21. http://dx.doi.org/10.1590/2177-9465-ean-2017-0208.

39. Alvis Zibran M, Mohammadnezhad M. Management of Type 2 Diabetes and Chronic Kidney Disease in Fiji in 2018: Knowledge, Attitude, and Practice of Patients. Rev. Diabet. Stud. 2019 [cited 2020 Mar 11]; 15:26-34. DOI: https://doi.org/10.1900/RDS.2019.15.26.

40. Oliveira KCSd, Zanetti ML. Knowledge and attitudes of patients with diabetes mellitus in a primary health care system. Revista da Escola de Enfermagem da USP. 2011 [cited 2020 Mar 11]; 45(4):862-8. DOI: https://doi.org/10.1590/S0080-62342011000400010.

41. De Carvalhotorres H, Nogueiracortez D, Reis IA. Education assessment in diabetes group in primary health care. Ciencia y Enfermería. 2016 [cited 2020 Mar 11]; 22:35-45. Available from: https://scielo.conicyt.cl/pdf/cienf/v22n3/0717-9553-cienf-2203-00035.pdf.

42. Vieira GdLC, Cecílio SG, Torres HdC. The perception of users with diabetes regarding a group education strategy for the promotion of self-care. Escola Anna Nery. 2017; 21(1). DOI: https://doi.org/10.5935/1414-8145.20170017.

43. Capellari C, Costa B, Larré A, Pasin D, Cardoso A, Vasconcelos L, et al. Educational Interventions and Glycemic Control: Integrative Review. Journal of Diabetes \& Metabolism. 2016 [cited 2020 Mar 11]; 7(7). DOI: https://doi.org/10.4172/21556156.1000675.

44. Dempster M, McCarthy T, Davies M. Psychological adjustment to Type 2 diabetes and relationship quality. Diabetic Medicine. 2011 [cited 2020 Mar 11]; 28(4):487-92. DOI: https://doi.org/10.1111/j.1464-5491.2010.03214.x.

45. Ghannadi S, Amouzegar A, Amiri P, Karbalaeifar R, Tahmasebinejad Z, Kazempour-Ardebili S. Evaluating the Effect of Knowledge, Attitude, and Practice on Self-Management in Type 2 Diabetic Patients on Dialysis. J Diabetes Res. 2016 [cited 2020 Mar 11]; 2016:3730875. DOI: https://doi.org/10.1155/2016/3730875.

46. Schinckus L, Dangoisse F, Van den Broucke S, Mikolajczak M. When knowing is not enough: Emotional distress and depression reduce the positive effects of health literacy on diabetes self-management. Patient Education and Counseling. 2018 [cited 2020 Mar 11]. DOI: https://doi.org/10.1016/j.pec.2017.08.006.

47. Wong FKY, Chow SKY, Chan TMF. Evaluation of a nurse-led disease management programme for chronic kidney disease: A randomized controlled trial. International Journal of Nursing Studies. 2017 [cited 2020 Mar 11]; 47(3):268-278. DOI: https://doi.org/10.1016/j.ijnurstu.2009.07.001. 